A NOTE ON EXACT TESTS FOR SERIAL CORRELATION

by

Christopher A. Sims

Discussion Paper No. 26, March 1973

\author{
Center for Economic Research \\ Department of Economics \\ University of Minnesota \\ Minneapolis, Minnesota 55455
}




\section{A NOTE ON EXACT TESTS FOR SERIAL CORRELATION}

by

Christopher A. Sims

\section{Introduction}

Durbin (1970) and Abrahamse and Koerts (1971) have proposed linear transforms of the OIS residral vector which give it a convenient covariance matrix. Though both proposed transforms achieve the same covariance matrix on the null hypothesis, -

I Both techniques allow specification of an arbitrary idempotent covariance matrix for the transformed residuals.

they differ in important respects.

Durbin presents his transform in easily computable form, exploiting standard statistics printed out in OLS computer programs, and his formulas involve matrix inversions of order no greater than $2 \mathrm{k}$, where $\mathrm{k}$ is the number of independent variables. On the other hand, Durbin makes no claims related to the power of tests based on his transformed residuals, though it is easy to see that his transformed residual vector is, like the origina] OLS residual vector, elementwise consistent as sample size expands.

Aorahamse and Koerts $(A-K)$ optimize the choice of linear transform of the OLS residuals, over a class of transforms including Durbin's, minimizing

$$
Q=E\left[\left(u-B^{\prime} \hat{u}\right) \cdot\left(u-B^{\prime} \hat{u}\right)\right],
$$

where $u$ is the true residual vector, $\hat{u}$ is the OLs estimate, and $B^{\prime} \hat{u}$ is the transformed vector. Minimizing $\cap$ under the null hypothesis is not the same thing as maximizing power of tests based on $B^{\prime} \hat{A}$ by an means; but for alternative hypotheses near $\mathrm{H}_{\mathrm{O}}$, minimizing $Q$ and maximizing power are probably closely 
related. On the other hand, $A-K^{\prime}$ s formula involves taking the square root of an $(n-k) \times(n-k)$ matrix, where $n$ is sample size, and therefore appears much less easy to implement than Durbin's formula.

In this note we will see that a) Durbin's transtorm behaves noticeably worse than $A-K^{\prime}$ 's in a certain important class of cases and b) Durbin's computational ideas can be applied to make A-K's transform nearly as simple as Durbin's own.

1. A special Case

Suppose we have the regression

1) $y=x_{1} \beta+u$. $n \times 1 n \times 1 \quad n \times 1$

Let $x_{1}=a z_{3}+z_{2}$,

where $z_{i_{1}}$ and $z_{z_{2}}$ are orthonormal vectors, and suppose we wish to transform the OIS residual vector $\hat{u}$ so it will have the same covariance matrix as residuals from an oLs regression of $u$ on $z_{2}$. The following paradoxes emerge from examining Durbin's procedure: A) Durbin's transform is different if we substitute $z_{2}^{*}=-z_{2}$ for $z_{2}$, so that $x_{1}=a z_{1}-z_{2}^{*}$, despite the fact that $z_{2}$ itself' is a "dumy" vector whose sign does not af'fect the problem; B) In the problem as first formulated, with 7, Durbin's transformed residuals do not approach $\hat{u}$ as $a \rightarrow 0$. Verification of these points is left to the reader. Neither of these two paradoxes arises for the $A-K$ transtorm. 
Paradox ( $B$ ) is disturbing, since it is often argued

$\checkmark$ I am not sure this argument is of much practical relevance, myself.

that in econometric practice we frequently meet cases where independent variables are close in mean square to low-order trigonometric polynomials. Generalizing the example to $k>1$, this suggests that small a's are likely in practice. It is known for this case that $\hat{u}$ itseif has near $l y$ the convenient covariance matrix the transforms aim to achieve. A- $K$ exploits this fact and leaves $\hat{u}$ nearly unchanged, while Durbin's transform may substantially alter $\hat{u}$, probably noticeably reducing nower in the process.

\section{Computing the Abrahamse and Koerts Transform}

In the $A-K$ notation, our problem is to find an $n \times n$ matrix 2 such that

e) $B^{\prime} B=0$,

3) $n \cdot x_{1}=0$,

and $\operatorname{tr} B$ is a maximum.

In Durbin's notation, $\sigma=I-x_{2}\left(x_{2} \cdot x_{2}\right)^{-1} x_{2}^{\prime},-/ x_{2}$ being $n \times k$.

- We ignore for the time being Durbin's $x_{3}$, which consists of variables included in the original regression and also in the cummy regression of $u$ on "convenient" variables.

$A-K$ begin by choosing $P$ an arbitrary $n \times(n-k)$ matrix of orthonormal eigenvectors of $M=I-X_{1}\left(X_{1} X_{3}\right)^{-1} X_{1}^{\prime}$ such that $P^{\prime}=M$, while $K$ is an arbitrary $n \times(n-k)$ matrix of orthonormal. eigenvectors of $n=I-X_{2}\left(X_{2}^{\prime} X_{2}\right)^{-1} X_{2}$ ' such that $K^{\prime}=0$. They then show that any $B$ satisfying the restrictions (2) and (3) 


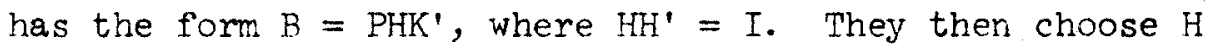
optimally.

The dif'iculty of A-K's suggested computations comes from choosing $K$ and $P$ too arbitrarily. Let $P_{3}$ be an $n \times(n-2 k)$ matrix of orthonormal vectors spanning the space orthogonal to both $X_{1}$ and $X_{2}$. Let $P_{1.2}$ span the subspace of the space spanned by $X_{1}$ and $X_{a}$ which is orthogonal to $X_{2}$. Let $P_{2.1}$ be defined analogously. Then we can take $P=\left[\mathrm{P}_{2.1}, \mathrm{P}_{3}\right]$, $K=\left[P_{1.2}, P_{3}\right]$

Applying $A-K^{\prime}$ 's procedure, we now find that $H$ has the form

$$
\mathrm{H}=\left[\begin{array}{ll}
\mathrm{H}_{\mathrm{k}} & 0 \\
\mathrm{O} & \mathrm{I}
\end{array}\right]
$$

where $H_{k}$ is $k \times k$. Using $A-K^{\prime}$ 's method for choosing $H_{k}$ and applying Turbin's computational ideas, we write

$$
\text { 4) } \begin{aligned}
\mathrm{z} \cdot \mathrm{y}=\hat{u}_{12}+\mathrm{X}_{1.2} \mathrm{G}_{12}\left(\mathrm{P}_{2}^{-1}\right)^{\prime}\left(\mathrm{P}_{2}^{-1} \mathrm{G}_{21} \mathrm{G}_{1}^{-1} \mathrm{G}_{12}\left(\mathrm{P}_{2}^{-1}\right)^{\prime}\right)^{-\frac{1}{2}} \\
\mathrm{P}_{2} \mathrm{X}_{2.1}^{\prime} \mathrm{y} .
\end{aligned}
$$

Here $\hat{A}_{12}$ is the residual vector from OIs regression of $y$ on $x_{1}$ and $x_{2}$ together. Following Durbin's notation, $x_{i \cdot j}$ is that part of $x_{i}$ not explained by linear regression on $x_{j}$, $G_{1}=\left(X_{1.2}{ }^{\prime} X_{1.2}\right)^{-1}, G_{2}=\left(X_{2.1} X_{2.1}\right)^{-1}, G_{12}=G_{21}{ }^{\prime}=G_{1} X_{1.2} x_{2.1} G_{2}$, and $P_{2}$ is any square matrix satisfying $P_{2} P_{2}^{\prime}=G_{2}$. The exponent for a positive def'inite matrix $Q=W W^{\prime}$, where $D$ is diagona 1 and $W W^{\prime}=I$, is defined by $O^{2}=N^{1} W^{\prime}$, where $D^{:=}$is the elementwise square root of $D . G_{1}$ and $G_{2}$ are, as Durbin pointed out, scalar multiples of the covariance matrices of $c_{1}$ and $c_{2}$, 
respectively, where $c_{1}$ and $c_{2}$ are the coefficients of $X_{1}$ and $x_{2}$ in the OIS estimate of the joint regression of $y$ on these two variables. $G_{12}$, which does not enter Durbin's formula, is proportional to the matrix of covariances between $c_{1}$ and $c_{\hat{c}}$. Finally, explicit computation of the vector $x_{2.1}$ can be avoided by noting that the term $\mathrm{P}_{2}^{\prime} \mathrm{X}_{2.1}^{1}{ }^{\mathrm{y}}$, appearing at the end of (4), can be rewritten in terms of $c_{2}$, to yield

5) $\mathrm{B}^{\prime} \mathrm{y}=\hat{u}_{12}+\mathrm{X}_{1.2} \mathrm{G}_{12}\left(\mathrm{P}_{2}^{-1}\right)^{\prime}\left(\mathrm{P}_{2}^{-1} \mathrm{G}_{21} G_{1}^{-1} G_{12}\left(\mathrm{P}_{2}^{-1}\right)^{\prime}\right)^{-t}$ $P^{-1} c_{2}$

Formula (5) requires taking one symmetric square root of a $k \times k$ matrix and inversion of the $k \times k$ matrix $G_{l}$, while avoiding the taking of a triangular square root of $G_{1}$ as is required for Durhin's transform. This increased computational requirement is modest, and for small $k$ it is negligible. For $k=1$ it is only a matter of finding the right sign for $x_{2}$. What if there is a set of variables $X_{3}$ included in both the origina? regression matrix and in the dumry regression? In tris case $P_{3}$ could not be chosen as $n \times(n-2 k)$. However, appropriate modification of formula (5) is simple. Now $\mathrm{x}_{1}$ becomes the matrix of variables appearing only in the original regression, $x_{?}$ the matrix of variables appearing on $7 y$ in the dummy regression. The auxiliary regression from which $G_{1}$, $\vec{c}_{2}, \hat{x}_{12}, c_{2}$, and $\hat{u}_{12}$ are drawn is now a joint regression on $x_{1}$, $x_{2}$, and $x_{3}$, and $x_{1.2}$ is replaced by $x_{1.23}$. 
REFERENCES

Durbin, J. (1970), "An Alternative to the Bounds Test for Serial Correlation in Least-Squares Regression", Econometrica, $38,422-429$.

Abrahamse, A.P.J. and J. Koerts (1971), "New Estimators of Disturbances in Regression Analysis", JASA, 66, 71-7't. 